\title{
VLBA imaging of radio-loud BAL QSOs
}

\author{
F.M. Montenegro Montes ${ }^{* a b c}$, K.-H. Mack, ${ }^{a}$ C.R. Benn, ${ }^{d}$ R. Carballo, ${ }^{e}$ D. Dallacasa, ${ }^{f a}$ \\ J.I. González-Serrano, ${ }^{g}$ J. Holt ${ }^{h}$ and F. Jiménez-Luján ${ }^{g i}$ \\ ${ }^{a}$ INAF - Istituto di Radioastronomia, Via P. Gobetti 101, I-40129 Bologna, Italy \\ ${ }^{b}$ Dpto. de Astrofísica, Universidad de La Laguna, Avda. Astrofísico Fco. Sánchez s/n, E-38209 \\ La Laguna, Spain \\ ${ }^{c}$ Instituto de Astrofísica de Canarias, C/ Via Láctea s/n, E-38200 Tenerife, Spain \\ ${ }^{d}$ Isaac Newton Group, Apartado 321, E-38700 Santa Cruz de La Palma, Spain \\ e Dpto. de Matemática Aplicada y Ciencias de la Computación, Univ, de Cantabria, ETS \\ Ingenieros de Caminos Canales y Puertos, \\ Avda. de los Castros s/n, E-39005 Santander, Spain \\ ${ }^{f}$ Dipartimento di Astronomia, Università di Bologna, Via Ranzani 1, I-40127 Bologna. Italy \\ ${ }^{g}$ Instituto de Física de Cantabria (CSIC-Universidad de Cantabria), Avda. de los Castros s/n, \\ E-39005 Santander, Spain \\ ${ }^{h}$ Leiden Observatory, Leiden University, P O Box 9513, NL-2300 RA Leiden, The Netherlands \\ ${ }^{i}$ Dpto. de Física Moderna, Univ. de Cantabria, Avda de los Castros s/n, E-39005 Santander, \\ Spain \\ E-mail: fmmeira.inaf.it
}

Broad Absorption Line Quasars (BAL QSOs) have been found to be associated with extremely compact radio sources. These reduced dimensions can be either due to projection effects or these objects might actually be intrinsically small. Exploring these two hypotheses is important to understand the nature and origin of the BAL phenomenon because orientation effects are an important discriminant between the different models proposed to explain this phenomenon. In this work we present VLBA observations of 5 BAL QSOs and discuss their pc-scale morphology.

The 9th European VLBI Network Symposium on The role of VLBI in the Golden Age for Radio Astronomy and EVN Users Meeting September 23-26, 2008

Bologna, Italy

\footnotetext{
* Speaker.
} 


\section{Introduction}

The presence of Broad Absorption Lines (BALs) in the optical spectra of some quasars is interpreted as a footprint of powerful quasar winds accelerated by the central engine up to velocities of $\sim 0.2$ c. Broad Absorption Line Quasars (BAL QSOs) have been revealed to be associated with extremely compact radio sources with projected linear sizes smaller than $1 \mathrm{kpc}$. To explain this, one possibility is that these could be intrinsically small objects but they might also be compact due to projection effects. The first hypothesis would find a natural explanation within the so-called evolutionary scenario proposed to interpret the nature of BAL QSOs. A second option would suggest that BAL QSOs could be oriented in a particular way, the basis of some orientation scenarios that explain in this way the detection of the BAL phenomenon in a subset of $15-20 \%$ of all QSOs.

In [1] it was shown that radio BAL QSOs share several properties with the class of GigahertzPeaked Spectrum (GPS) sources, which are radio sources going through the first stages of their evolution. VLBI observations are crucial to reveal the pc-scale structure of these objects and help understand their geometrical disposition. For this purpose we present here two-frequency VLBA observations of 5 radio BAL QSOs.

\section{Sample and observations}

The list of observed objects can be found in Table 1, with columns being the observation date, radio coordinates in $\mathrm{J} 2000$, redshift and flux densities at 4.8 and $8.3 \mathrm{GHz}$ from previous continuum Effelsberg observations. Four of the BAL QSOs belong to the sample presented in [1], and we have also observed the radio BAL QSO $1537+58$. Details on the radio spectra and polarisation properties of these objects can be found in [1].

Table 1: List of 5 BAL QSOs observed with VLBA

\begin{tabular}{crrrccc}
\hline ID & Obs. Date & $\begin{array}{r}\text { RA } \\
(\mathrm{J} 2000)\end{array}$ & $\begin{array}{r}\text { DEC } \\
(\mathrm{J} 2000)\end{array}$ & $\mathrm{z}$ & $\begin{array}{c}\mathrm{S}_{4.8 \mathrm{GHz}} \\
(\mathrm{mJy})\end{array}$ & $\begin{array}{c}\mathrm{S}_{8.3 \mathrm{GHz}} \\
(\mathrm{mJy})\end{array}$ \\
\hline $0135-02$ & $21-10-2007$ & 013515.243 & -021349.37 & 1.820 & $27.2 \pm 0.6$ & $31.9 \pm 0.8$ \\
$0837+36$ & $06-11-2007$ & 083745.589 & +364145.60 & 3.416 & $30.2 \pm 2.3$ & $12.1 \pm 0.8$ \\
$1159+01$ & $23-12-2007$ & 115944.827 & +011206.98 & 1.989 & $137.8 \pm 1.7$ & $158.0 \pm 2.0$ \\
$1537+58$ & $25-11-2007$ & 153729.549 & +583224.80 & 3.059 & $26.5 \pm 0.4$ & $40.6 \pm 0.8$ \\
$1624+37$ & $23-01-2005$ & 162453.481 & +375806.66 & 3.377 & $23.3 \pm 1.1$ & $15.0 \pm 0.1$ \\
\hline
\end{tabular}

Two receivers were used, centred on the $\mathrm{C}$ and $\mathrm{X}$ bands (at about 5.0 and $8.4 \mathrm{GHz}$ respectively). A minimum of two frequencies allows us to have different resolutions to look for individual components and to study the spectral indices and the polarisation properties of sub-components. Details on the observational setup, calibration, imaging and further analysis of the VLBA data will be presented in a forthcoming paper currently in preparation.

\section{VLBA maps and discussion}

The VLBA maps of BAL QSOs 0135-02 and 0837+37 are shown in Figure 1, where the peak flux densities are also noted. These two sources are unresolved with the VLA at $8.4 \mathrm{GHz}$, and they 
still appear point-like at the milliarcsecond-scale. Gaussian fits at $8.4 \mathrm{GHz}$ give angular extensions of $3.5 \times 0.9$ mas and $2.3 \times 1.0$ mas for $0135-02$ and $0837+37$, respectively. These translate into linear dimensions of about $30 \times 8 \mathrm{pc}$ and $19 \times 8 \mathrm{pc}$ for these two sources, respectively.
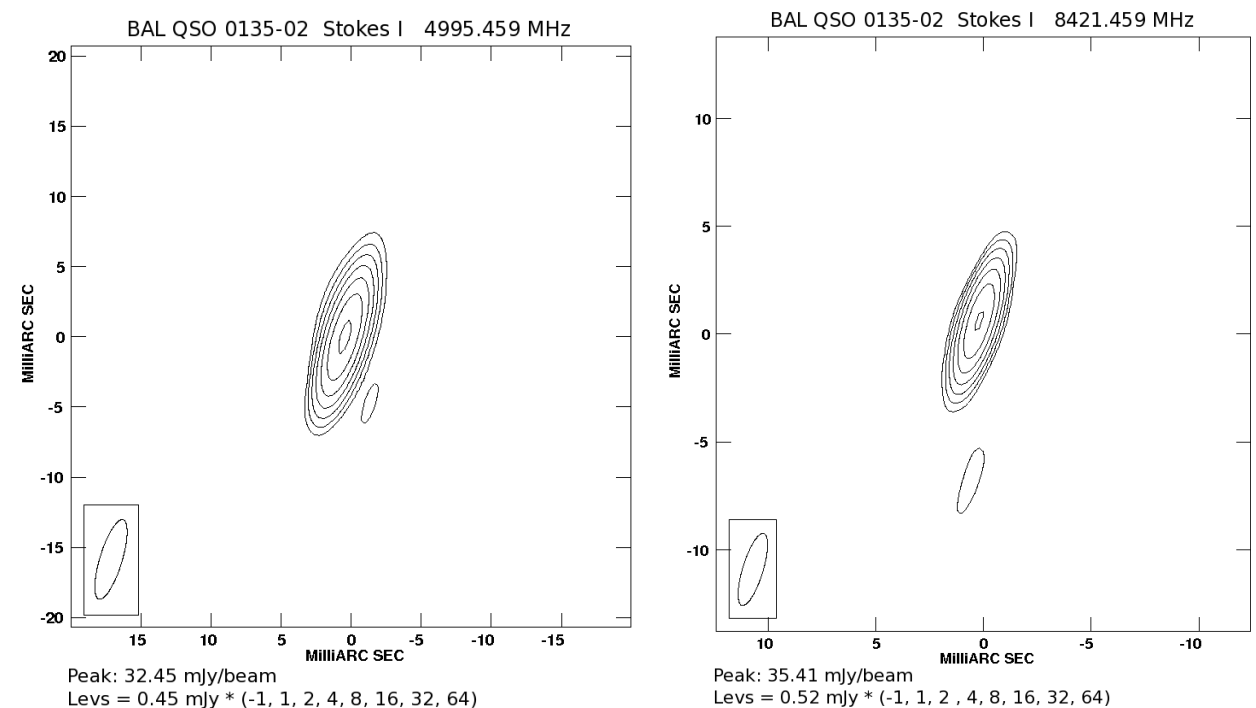

Peak: $35.41 \mathrm{~mJ} / \mathrm{beam}$
Levs $=0.52 \mathrm{mJy} *(-1,1,2,4,8,16,32,64)$
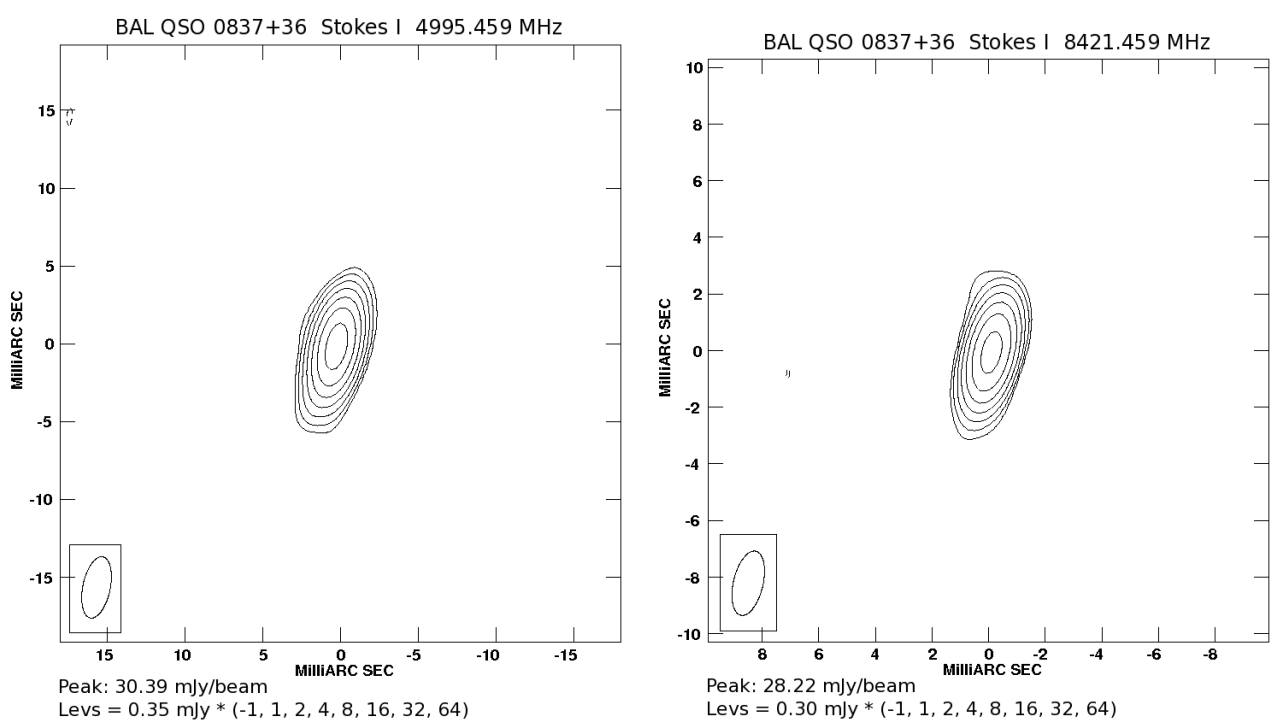

Figure 1: VLBA maps at $5 \mathrm{GHz}$ (left) and $8 \mathrm{GHz}$ (right) showing the pc-scale structure of BAL QSOs 0135-02 (top) and 0837+36 (bottom). The synthesised beam size is shown in the lower left corner of the map. Contours are in logarithmic scale according to the legend.

When compared to our previous Effelsberg and VLA measurements, there are slight increases of about $20 \%$ and $10 \%$ in the flux densities of $0135-02$, at 5.0 and $8.4 \mathrm{GHz}$, respectively. This might suggest moderate variability, and probably excludes the possibility of a fainter extended component not recovered in the VLBA maps. However, for $0837+36$, the $8.4-\mathrm{GHz}$ flux density would have increased about $130 \%$ in 2.3 years (observer's time), with a variability significance $\sigma_{\text {Var }}$ 
$=\left(S_{2}-S_{1}\right) / \sqrt{\sigma_{1}^{2}+\sigma_{2}^{2}}=5.2$, while at $5.0 \mathrm{GHz}$ both the Effelsberg and the VLBA measurements give similar values. If the variability at $8.4 \mathrm{GHz}$ is real and not due to a calibration error, the radio spectrum of this source has considerably flattened between these two frequencies.
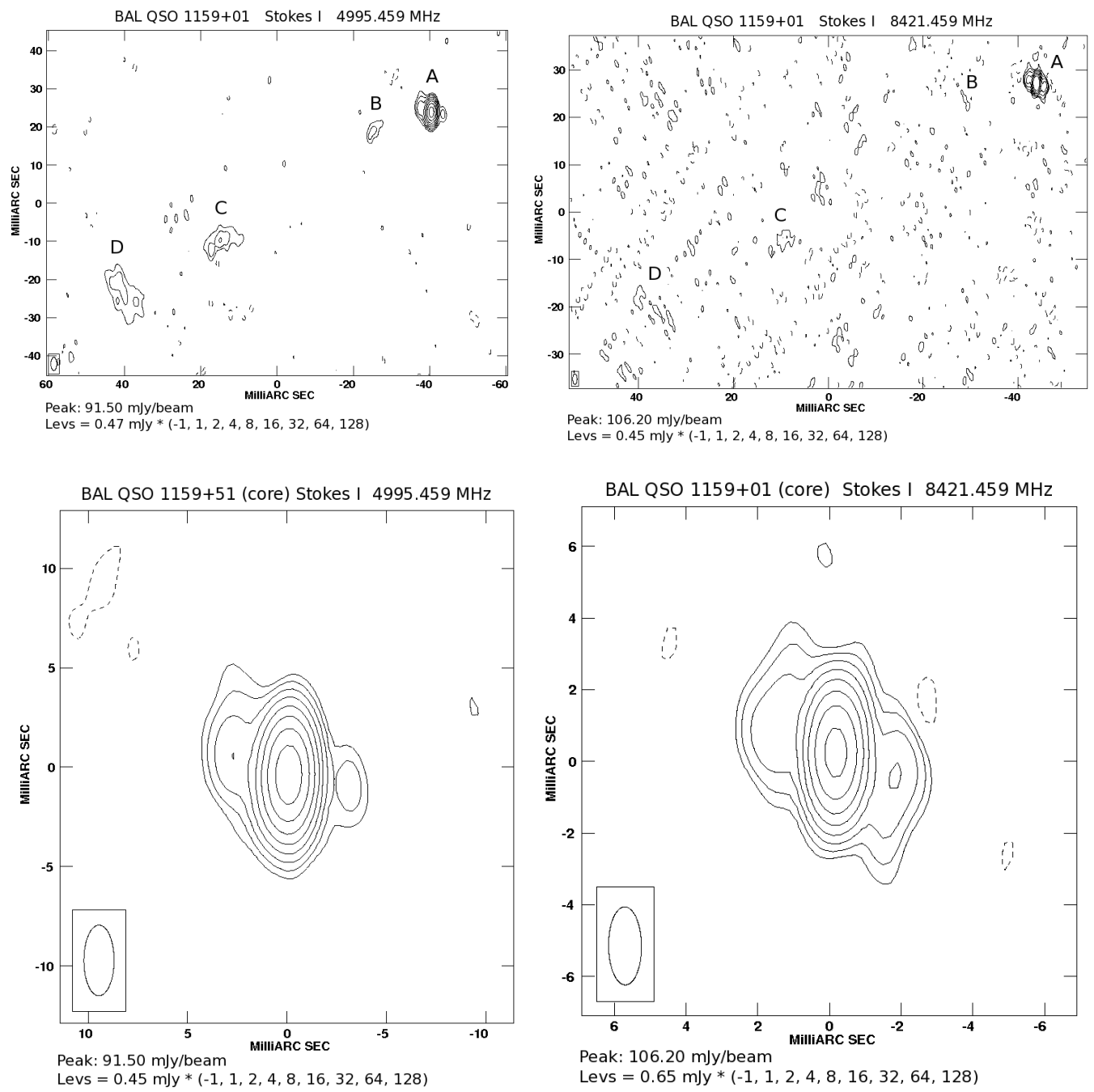

Figure 2: VLBA maps at $5 \mathrm{GHz}$ (left) and $8 \mathrm{GHz}$ (right) of BAL QSO 1159+01. The upper plots show the largest scale structure, while the lower plots detail the nuclear region. The synthesised beam size is shown in the lower left corner of the map. Contours are in logarithmic scale according to the legend.

In Figure 2 we see the extended structure of the interesting radio-loud BAL QSO 1159+01. A compact and bright component (A) can be seen associated with the radio core and also some faint extended emission which extends about 100 mas towards the SE from the central core. The faintness of this emission and the absence of an active hot-spot suggest this to be the remainder of a jet that has ceased its activity. An estimate of the extension of the diffuse emission has been determined measuring the distance between the A and D components, yielding 100 mas, which corresponds to a projected linear size of $0.85 \mathrm{kpc}$. If there is no significant variability, no additional 

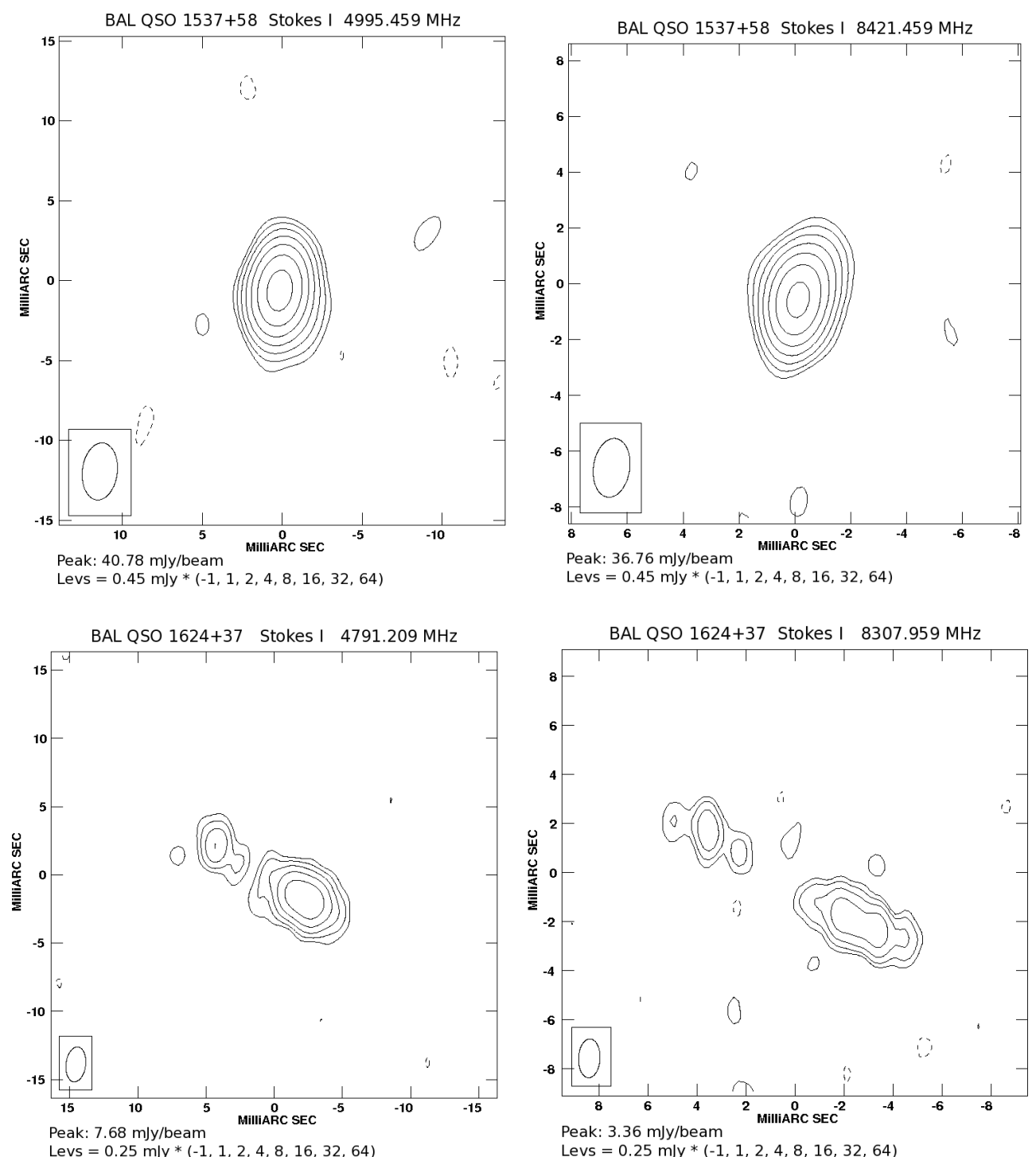

Figure 3: VLBA maps at $5 \mathrm{GHz}$ (top) and $8 \mathrm{GHz}$ (bottom) showing the pc-scale structure of BAL QSO 013502. The synthesised beam size is shown in the lower left corner of the map. Contours are in logarithmic scale according to the legend.

flux density is expected at longer distances from the core because the previously measured Effelsberg flux density is completely recovered when adding up the flux densities of the 4 components (A to D). The faintness of components B, C and D in the 8.4-GHz map is consistent with a steep spectral index, which is expected if the emission comes from relative relaxed particles in a nonactive region. Also at this frequency the summed flux density from all 4 components approximately equals the flux density from the VLA maps.

Another remarkable characteristic of $1159+51$ is the presence of two symmetric extensions 
very close to the central core (see the bottom of Figure 2). At both 5.0 and $8.4 \mathrm{GHz}$ a central brighter core and two symmetric components at about 2 arcsec from the centre can be seen. One of them is located towards the NWW and the other in the opposite direction. The first one is slightly brighter than the other at $5 \mathrm{GHz}$, but at $8 \mathrm{GHz}$ they have similar flux densities as can be seen in the contour map. This might be the origin of a new jet coming from the central nucleus. These two jets would not be aligned but both directions form an angle of about $50^{\circ}$.

Finally, Figure 3 shows the VLBA maps of $1537+58$ and $1624+37$. The first one looks quite compact and is just barely resolved at both frequencies. As in the cases of $0135-02$ and $0837+36$ some variability can reveal signs of beaming, with a flux density increase of $\sim 50 \%$ at $5 \mathrm{GHz}$ and $\sigma_{V a r}=3.5$. The unusual BAL QSO $1624+37$ shows a core-jet structure clearly visible at both frequencies. There is a more compact component (core) with flat spectral index and a more extended component towards the SE direction (jet) with steeper spectral index. The separation between the emission peaks of the two components is 8 mas. The core seems to have a very faint component at 1-2 mas towards the jet direction that could be the emission from the base of the jet.

\section{Conclusions}

Our contribution increases the number of VLBI observations of BAL QSOs published in the literature. A total of 17 of these sources can be found combining this work with [2], [3] and [4]. A variety of geometries are found among these sources: 7/17 are unresolved with VLBI baselines, 7/17 show a core-jet structure and 3/17 show a central bright core and two fainter extensions in approximately opposite directions, similar to Compact Symmetric Objects (CSOs). This variety of geometry is difficult to reconcile with simple orientation scenarios trying to explain the nature of BAL QSOs.

\section{References}

[1] F.M. Montenegro-Montes, K.-H. Mack, M. Vigotti, C.R. Benn, R. Carballo, J.I González-Serrano, J. Holt and F. Jiménez-Luján, Radio spectra and polarisation properties of radio-loud Broad Absorption Line Quasars, MNRAS 388, 1853 (2008) [arXiv: 0805 . 4746 ].

[2] D.R. Jiang and T.G. Wuang, EVN Observations of three Broad Absorption Line Quasars, A\&A 397 L13 (2003).

[3] M. Kunert-Bajraszewska and A. Marecki, FIRST-based survey of CSS sources ( V. Milliarcsecond-scale morphology of CSS sources), A\&A 469, 437 (2007) [arXiv : 0704 . 0351 ].

[4] Y. Liu, D.R. Jiang, T.G. Wang and F.G. Xie, The compact structure of radio-loud broad absorption line quasars, MNRAS 391, 246 (2008) [arXiv: 0808 . 2105]. 